\title{
Gitans et jeunes de «bonnes familles » dans les trafics de drogues
}

\section{Lamia Missaoui}

\section{(2) OpenEdition \\ 1 Journals}

Édition électronique

URL : https://journals.openedition.org/remi/1618

DOI : $10.4000 /$ remi.1618

ISSN : $1777-5418$

Éditeur

Université de Poitiers

\section{Édition imprimée}

Date de publication : 12 décembre 2002

Pagination : 77-92

ISBN : 2-911627-32-6

ISSN : 0765-0752

Référence électronique

Lamia Missaoui, "Gitans et jeunes de «bonnes familles » dans les trafics de drogues », Revue européenne des migrations internationales [En ligne], vol. 18 - n³| 2002, mis en ligne le 09 juin 2006, consulté le 15 avril 2022. URL : http://journals.openedition.org/remi/1618 ; DOI : https://doi.org/ 10.4000/remi. 1618

Ce document a été généré automatiquement le 15 avril 2022.

(C) Université de Poitiers 


\title{
Gitans et jeunes de « bonnes familles » dans les trafics de drogues
}

\author{
Lamia Missaoui
}

Une recherche précédente, concernant les Gitans avait attiré mon attention sur les trafics de psychotropes "durs " caractérisant diverses populations de la frontière franco-espagnole en bordure méditerranéenne (Missaoui, 1999). Quelques constats sur l'apparition dans ces trafics de jeunes issus des "honorables familles locales", me permirent d'organiser une recherche en direction de cette population qui vient de l'intérieur même de nos sociétés, faisant collectif autour de la production de normes originales, dérogatoires des reproductions sociales.

Depuis 1996 l'implication dans des trafics de drogue de jeunes gens, non Gitans, appartenant à des familles de divers milieux perpignanais, toulousains et barcelonais, présentes depuis plusieurs générations dans ces régions se développe dans des quartiers à forte concentration gitane. C'est là que se fournissent ces jeunes en rupture de continuités familiales : ironie de l'histoire, les «ethniques", les Gitans, qui furent associés aux consommations d'héroïne par des « jeunes de bonne famille » au début des années $80^{1}$, « sous-traitent » à leur tour des jeunes de ces milieux dits « bourgeois ».

L'apparition de ces nouveaux acteurs dans le paysage complexe des économies de psychotropes suggérait donc un changement de forme sociale ${ }^{2}$, impliquant les interactions généralisées entre groupes et comportements collectifs déviants. Dans cet article, nous voudrions comprendre comment l'étranger de l'intérieur (Missaoui, 2000), le Gitan, est devenu une ressource pour ces jeunes, quelles interactions sont apparues qui ont réduit, voire annulé la distance sociale historiquement instituée ? quelles occasions, quelles situations ont pu permettre ces rapprochements? Les jeunes de «bonnes familles » sont-ils devenus eux-mêmes des « étrangers de l'intérieur »? qu'en est-il de ces "mauvais héritiers" ? Sont-ils des «Déviants occasionnels", saisis dans une phase réversible d'un voyage initiatique? «étrangers de l'intérieur»? N’y a-t-il pas là occasion de brouiller les cartes de ces catégories construites par les théories de l'ethnicité. Notre recherche nous conduisait à envisager des actions partagées entre ces «ethniques" gitans et ceux que nombre de chercheurs n'envisagent même pas de 
désigner comme tels, parce que ce sont des gens «bien d'ici» ou encore les "gens bien " d'ici. Mais alors comment rendre compte de ce qui se transforme dans ces pratiques communes, illégales et illicites?

Du visible à l'invisible

La présence de ces jeunes est difficilement repérable sur les lieux de trafics connus parce que discrète. «Tu sais, on n'est pas les seules sur ces trafics, on nous remarque nous parce qu'on est mal organisés, mais d'autres plus jeunes, plus d'ici, ne se font jamais arrêter... Ne t'arrête pas au nombre de Gitans qui attendent dans les tribunaux pour être jugés... » (Carlos, Gitan de Barcelone).

5 C'est donc à l'extérieur des lieux « marqués » que ces « enfants honorables » trafiquent. Comment repérer, décrire et définir ces initiatives, ces échanges entre ces jeunes et les Gitans si les manifestations de leurs interactions ne sont pas observables dans les territoires classiques support aux trafics illicites? Pour l'objet de la recherche qui nous mobilise, la compréhension se situe dans l'analyse de l'inter-ethnicité (Hily, 2001) et non seulement au cœur des groupes ou des communautés ${ }^{3}$. C'est donc à l'intersection entre ces groupes marqués par la désignation "ethnique " (et pour nous l'autochtone représenté par les jeunes de «bonne famille » est aussi ethnique) que nous trouvons des réponses.

6 L'appel aux propositions de Barth (Poutignat et Streiff, 1995), qui signalent l'ethnicité dans des situations mettant en scène, généralement dans l'espace public, des façons d'être caractéristiques de tel ou tel groupe ethnique ${ }^{4}$, ne nous permet pas d'envisager ces réciprocités et métissages, essentiellement visible à partir de ces zones intermédiaires qui sont justement dans les intersections. Car ce sont bien ces interpénétrations de manières d'être qui contribuent à produire une situation où les échanges sont possibles. Non seulement un groupe s'appuie, pour instaurer et élargir ses activités, sur l'autre mais plus encore ces $P^{2} i_{0}{ }^{5}$, les mieux disposés, dans leurs milieux familiaux, à répéter les conformités normatives, empruntent à l'« étranger gitan » savoir-faire et connaissances du fonctionnement des comportements sociaux. Les circonstances, les moments et les situations où «pactisent ", passent parole, des populations différentes, afin de produire des codes d'honneur commun permettent de saisir ce qui se trouve modifié du rapport à l'altérité.

Lieux de commercialisation

7 Il suffirait de dire à quiconque se renseignerait sur les lieux "classiques » de commercialisation de l'héroïne, de la cocaïne, et d'autres psychotropes d'usage illicite, de se rendre autour de monuments : cathédrales, châteaux ou ouvrages prestigieux. Il s'agirait alors de se rapprocher de ces édifices remarquables pour rencontrer brusquement, en une concentration de misère, d'identités ethniques variées (et bien sûr, répétons le, pour nous l'autochtone et le touriste bien plus intéressés par des transactions dans des couloirs obscurs que par l'admiration des édifices historiques sont aussi des ethniques) de voitures de police tournant sans cesse, de prostitué(e)s visiblement atteint(e)s par l'usage des psychotropes.

Quant aux lieux de première concentration des produits, voire de production, un passage par les zones périurbaines habitées essentiellement par des Gitans y conduit inévitablement.

9 Certains centres urbains et périurbains particulièrement stigmatisés accueillent comme partout les migrants les plus démunis ou enferment les étrangers de l'intérieur; pauvreté du foncier se conjugue avec pauvreté économique et désintérêt des élus 
locaux. Ces emplacements si facilement identifiables, et parfois observables (Bouhnik, 1994) posent cependant problème à ces jeunes trafiquants de "bonne famille » peu habitués à ces «décors ». Quand ils se rendent dans un quartier gitan de périphérie, c'est qu'on les connaît sinon ils le contournent en voiture : quelques arrêts brefs dans ces itinéraires et bientôt les fournisseurs arrivent. Transactions rapides, directes: le vendeur fixe alors un rendez-vous secondaire en dehors du quartier pour faire affaire. Dans les centres urbains, les passages de nos jeunes sont tout aussi rapides, ils ne s'attardent pas :

«Au début j'allais à Lérida, au plus direct, sous l'entrée de la cathédrale et du château maure, sur la colline du centre. Tu montes des marches avec des bancs de chaque côté : tous les allumés sont là, jeunes, vieux, filles, garçons, Gitans, Arabes, Noirs, Espagnols, avec des têtes rouges et des yeux comme des soucoupes. C'est la coke. Peut-être le crack pour l'un ou l'autre; donc l'héro. n'est pas loin. Les Gitanes se tiennent mieux, elles te demandent une cigarette et te proposent toutes les dopes. (...) Alors quand tu es passé une ou deux fois pour acheter, je te dis pas, on te repère à cent mètres... ".

10 Le problème est symétrique pour les dealers qui désirent sortir de leur quartier gitan pour vendre à ces nouveaux et si différents trafiquants ou fournir la «marchandise " dans ces quartiers de centre ville: la police qui y tourne sans cesse ne s'arrête pas devant le spectacle mais aperçoit immédiatement le nouveau venu différent dont la présence risque de signaler celle du dealer.

11 Un Gitan nous déclarera :

«Le plus difficile ç'a été de rencontrer tranquillement ces Paios. Ils venaient pas là pour acheter une dose mais pour se remplir les poches de "dineros"; c'étaient pas les voyous qu'on connaît bien, de Valencia, Saragosse ou Béziers et qui ont tous les mêmes looks [...]. Ils étaient prudents et pas flambeurs ».

12 À Tarragone à Gérone ou à Lerida, les mises en scènes urbaines de ces trafics sont les mêmes: un lieu de centralité près de la cathédrale qui coiffe la colline du centre historique, des bars et des personnages interlopes, les mêmes postures de prostitué(e)s ou de toxicomanes "bourrés ", dodelinant sur l'arrête d'un trottoir, des petits groupes de dealers accroupis, le passage furtif de clients des divers échanges proposés là, et le ballet des voitures de police.

Nouveaux trafiquants, nouveaux usages

La caractéristique des "fils de famille » était de ne pas se commettre sur ces scènes, connues par tous, et que nous venons de décrire.

Notre population, de jeunes de «bonnes familles", trafiquants non-consommateurs, était ailleurs. D'une part, elle était apparue dans des commerces transfrontaliers depuis peu, en prenant distance par rapport au traditionnel centre unique qu'était Barcelone, comme à la suite d'une opération concertée de délocalisation, et d'autre part elle se déployait dans des espaces discrets, obscurs et "propres ", " lisses », qui ne retenaient aucun regard curieux, comme ces jeunes eux-mêmes n'attiraient aucune attention particulière ; des «messieurs tout le monde » appliqués aux trafics de produits mortels. Comment cette transformation de mise en scènes urbaines tellement connues s'étaitelle opérée ? Comment avaient donc pu prendre place, dans ces échanges ces nouveaux trafiquants perpignanais ou espagnols? Comment ces nouveaux espaces des trafics purent-ils prendre place, entre ceux de la déshérence des camps gitans et ceux de la dégradation d'espaces centre urbains ? Comment les Gitans réussissaient-ils désormais à instituer et gérer des commerces constants et intenses avec des «Paios»? 
L'hypothèse de l'apparition de nouveaux réseaux liés à de nouvelles filières ne tenait pas : les mêmes Gitans qui avaient provoqué les excès d'autoconsommation dans leurs communautés, par impossibilité d'écouler ailleurs l'héroïne, tenaient ce nouveau marché. En fait la réponse est complexe et doit en premier lieu envisager d'importants remaniements des modes de production de l'héroïne, de diffusion des divers psychotropes et de commercialisation de produits médicamenteux (Ehrenberg, 1998), en cours à Barcelone et dans sa région, dans le sens d'une plus grande efficience commerciale, de la satisfaction d'une clientèle de plus en plus nombreuse et diversifiée quant à ses goûts et à ses moyens ${ }^{6}$.

Dans le département frontalier des Pyrénées-orientales, les Gitans andalous, regroupés dans un quartier excentré de Perpignan, contrôlaient déjà une partie des trafics, en particulier en direction des Gitans catalans, plus consommateurs que dealers de quelque envergure. D'ailleurs, "victimes " des accords entre dealers barcelonais et Gitans andalous, les Catalans furent refoulés de leurs lieux d'achat à Gérone et à Barcelone en quelques mois. Seule une petite ville espagnole frontalière, Figuères, échappa le long de l'année aux partages de marché. La côte proche, la "Costa Brava ", qui voit passer et résider des centaines de milliers de touristes en été (Cadaquès, Roses, etc.) possède un statut «spécial» en regard de ces partages : hors saison estivale, les réseaux "andalous» couvrent les besoins des populations résidentes et les habituels dealers qualifiés de "voyous » distribuent dans quelques boîtes encore ouvertes. Par contre en été, les Gitans andalous, et leurs obligés nouveaux trafiquants, se retirent de l'ensemble de la zone côtière sur une profondeur de dix à quinze kilomètres, c'est-àdire hors de la zone de grande concentration touristique.

Les Andalous facilitaient les transports de cocaïne et d'héroïne vers des destinations plus lointaines.

"C'était trop connu, trop fliqué, plein de balances : les Gitans espagnols ont eu raison de repartir à zéro avec des gars non-consommateurs, jamais repérés, et non usagers de ces boîtes. D'un côté les voyous nous couvraient, on ne voyait qu'eux, et de l'autre les Gitans du coin [les Catalans] nous couvraient aussi : ils trafiquaient très peu, bien moins que nous, ou que les voyous du pays, bien sûr, mais la vieille haine des habitants du coin en faisait les hommes de tous les dangers ; ça c'est une vieille histoire locale, voleurs de poules quand les gens avaient faim, voleurs d'enfants toujours et maintenant pourvoyeurs de tous les dangers. On leur colle tout sur le dos pour mieux se cacher " nous dit un jeune trafiquant, étudiant originaire d'un village rural.

17 Les jeunes «de familles» ne furent pas sollicités dans un premier temps, mais, par l'intermédiaire de Gitans catalans d'un certain âge, bien installés dans Perpignan et dans des villages de la vallée de la Têt, ce furent des personnes à maturité (nous avons pu en identifier trois de 51, 55 et 59 ans) les premières associées aux trafics. Les Gitans catalans ne jouèrent en somme qu'un rôle d'entremetteurs: ils signalaient aux Andalous des personnes bien connues, bien insérées dans les villages et en situation de grande difficulté économique ; dans les trois cas identifiés, ces personnes avaient connu un problème judiciaire antérieur (délits) qui les avaient déstabilisés professionnellement mais n'avait pas réellement atteint leur insertion sociale locale. Des personnes en somme victimes d'un «incident de parcours» dont le profil permettait de faire un "job de trafiquant", défini comme substitut à un impossible emploi, et qui exigeait discrétion et apparence de maintien dans les normes sociales locales. Deux sur trois trouvèrent des métiers non qualifiés impliquant de fortes mobilités, ce qui facilita leur activité de trafiquants. Ce profil, toutefois, s'il permettait 
une dissimulation rare jusque-là, était peu susceptible d'une grande immersion parmi les populations locales de consommateurs de psychotropes et encore moins d'influence sur des jeunes :

"C'étaient les "pépés tranquilles" du trafic. Les Gitans étaient bouche bée de constater que leurs premiers véritables "sous-traitants" Paios dissimulaient à ce point leurs activités et étaient capables de cette double façade : trafiquant de dure et homme respecté au village [...] » (Un jeune trafiquant perpignanais).

C'est en 1996, qu'une famille de Gitans andalous, implantée à Barcelone, à Gérone, Figuères, Perpignan et Béziers, commença à recruter des jeunes gens en mal de reproduction des situations familiales antérieures ou de financements pour des réussites professionnelles dans l'officialité. Les trois premiers dealers de "bonnes familles» furent des étudiants toulousains, d'écoles d'ingénieurs, un couple et un célibataire.

« Il a d'abord présenté le couple d'étudiants à X., du village de $\mathrm{Y}$ dans les Pyrénéesorientales. Ils se sont bien entendus. X. leur a montré les combines, comment attendre, comment se renseigner pour savoir si on passe par là ou ailleurs. Les Gitans ont vite compris leur chance : des "blancs" à leur merci, et des "bons blancs", les plus loin de leur façon de vivre, les modèles qu'on approche jamais, dans leur tête: tu vois ça, un étudiant futur ingénieur, de Toulouse, futur pharmacien de Montpellier, un fils à papa qui tiendra bientôt une belle boutique, un jeune paysan qui va acheter des terres. Et surtout des gars jeunes qui ont de l'influence sur d'autres jeunes comme eux, qui peuvent ouvrir un marché inaccessible pour tous les autres trafiquants et, comme dernier plus, qui sont bien placés, par leur âge pour fourguer toutes les occases, ecstasy et compagnie [...]» (couple de jeunes trafiquants à Lérida).

Dans deux cas, à notre connaissance, les diverses générations d'une même famille se mobilisent autour des trafics :

« Dans la famille L., la grand-mère vendait les "parachutes" d'héroïne en les tirant d'un sein et mettait l'argent dans l'autre sein ; le grand père trouvait les planques dans les vignes, dans les "casots" [petites granges agricoles, dans les champs]; les petits enfants allaient y chercher la dope quand nécessaire; la génération intermédiaire allait à Lérida, et les femmes vendaient des produits qui justifiaient les passages des clients. Les clients commandaient par téléphone tel ou tel produit, qui correspondait à telle ou telle drogue, car ils offraient tout ce qui était demandé sur le marché » (Un policier).

Dans leur mode de vie rien ne distinguait cette famille de ses voisins, sinon que, comme nous l'expliqua l'un d'eux :

«Il y a dix ans environ, un fils qui s'était encanaillé a tué un voyou; sa condamnation n'a pas été trop lourde, et ici on considérait que toute la famille s'était mise à le réintégrer; ils donnaient le spectacle d'une famille dynamique, qui a franchi un mauvais passage ${ }^{7} »$.

21 L'impression que ces «emplois " sont devenus «normaux " domine dès lors que des justifications sont avancées. Ces trafiquants ont le sentiment de gérer « un vice très répandu ", sans scandales ni violences, à la différence des voyous de milieux interlopes. Relations intra-familiales, relations interethniques et trafics : savoir « entrer-sortir » Parmi les 79 personnes ${ }^{8}$ que nous avons reconnues mobilisées par ces trafics de part et d'autre de la frontière catalane franco-espagnole, 17 étaient inconnues des services de police; pour les 62 restantes, les situations au moment de l'enquête allaient de l'emprisonnement à la mise en garde verbale par les policiers ou les gendarmes ou /et 
aux fouilles systématiques lors des passages de frontière et dans des situations parfois inattendues en ville :

Évitement des sanctions pénales et cohésions familiales

«On bénéficie d'un "bonus" auprès des juges et des policiers : les trafiquants nonconsommateurs qui sont dans notre cas, c'est-à-dire qui vivent toujours très "tranquillement" dans des familles de bonne réputation sont considérés comme rapidement amendables; alors on est prévenus avant d'être vraiment inquiétés : «votre fils file du mauvais coton», «on a des renseignements qui disent que...»; d'autres fois, tu circules tranquillement en ville et, ça m'est arrivé deux fois, une voiture te double et te fait signe de te garer: fouille, menaces, «dernier avertissement» des policiers; alors on sait exactement où on en est, si on peut encore continuer, s'il faut "rentrer dans le rang" quelque temps ou définitivement; mais il y a aussi les douaniers et les gendarmes; avec eux ça se passe pas de la même façon : direct la prison. Et puis on retrouve les juges, et la famille, donc une "bonne représentation", comme ils disent. Alors il faut arrêter et partir d'une façon ou d'une autre. Pour moi, quand ça s'est produit, ç'a été l'occasion d'en sortir, d'en finir [...]. Depuis le début, je me disais que c'était chaque fois, à chaque passage, fini ; alors, cette fin c'est affaire d'occasion. [...] Non, les Gitans t'embêtent pas si tu arrêtes. Mais peut-être à condition de ne plus rester là où on habite [...]. On n'arrête pas de savoir si on reste dans ce boulot, si on le quitte, si on y retourne, si on revoit ou non les Gitans, la famille, si on devient un voyou, on n'en finit pas de savoir où on est, ce que l'on quitte, ce que l'on trouve. C'est comme si tu avais le choix entre une foule de maisons avec autant de familles, où tu peux entrer et sortir comme tu le veux : tu te dis, "il faudra bien que j'en choisisse une un jour", parce que c'est très fatigant de changer sans arrêt de milieu, mais, c'est en même temps tellement agréable de vivre toutes ces facettes qu'il faut que quelque chose, quelqu'un t'arrête, te dise, "ça va maintenant, stop, tu restes ici". Ce qui est certain, c'est que les Gitans ne nous bloquent pas. On paye toujours la came à la livraison et on trouve forcément quelqu'un à mettre dans le coup. Alors, les Gitans, c'est "ni vu ni connu"; tu les croises un mois après et ils te voient même plus. C'est leur façon d'être correct; et moi je trouve que c'est très généreux: ils nous rendent à notre anonymat » (ancien trafiquant, Perpignan).

Les premiers résultats de l'enquête montrent que la grande majorité des familles de ces jeunes trafiquants présentent tous les caractères de cohésion. La qualité des rapports intra-familiaux étant précisément ce qui prévaut dans le recrutement de ces jeunes trafiquants. Nous supposions, en début d'enquête, que nous avions affaire à une population de jeunes de milieux particulièrement favorisés économiquement. Nous pensions que des jeunes ne pouvant reproduire les situations avantageuses de leurs parents fortunés choisissaient aujourd'hui ces activités comme autrefois les mêmes choisissaient le métier d'antiquaire et les échanges troubles qui le caractérisent: ces cas se présentaient, mais d'autres aussi, tels des jeunes de milieux agricoles modestes, ou encore des enfants de fonctionnaires de classes moyennes.

Nous présentons ci-dessous un tableau répertoriant la dispersion de notre échantillon de 79 personnes en France et Espagne selon les professions des parents, le nombre de générations de présence dans l'actuel lieu de résidence, le nombre de divorces parentaux, le niveau d'études des jeunes trafiquants et la localisation de leur résidence par rapport à celle de leurs parents. 


\begin{tabular}{|c|c|c|c|c|c|}
\hline Profession des parents & Effectifs & \begin{tabular}{|c|}
$\begin{array}{c}\text { Antériorité locale } \\
\text { (en générations) }\end{array}$ \\
\end{tabular} & $\begin{array}{c}\text { Divorces } \\
\text { des parents }\end{array}$ & Niveau études & $\begin{array}{l}\text { Lieu de } \\
\text { résidence }\end{array}$ \\
\hline \multirow[t]{3}{*}{$\begin{array}{l}\text { Ouvrier employé en } \\
\text { milieu rural }\end{array}$} & 7 & de $3 a 6$ & 0 & 2 bac & 4 dans famille \\
\hline & & & & 4 bact +2 & 2 près famille \\
\hline & & & & 1 bact 4 & 1 loin famille \\
\hline \multirow[t]{3}{*}{$\begin{array}{l}\text { Petite exploitation } \\
\text { agricole familiale }\end{array}$} & 5 & de $5 \mathrm{a}+$ de 10 & 0 & 3 bac & 3 dans famillc \\
\hline & & & & 1 bact 2 & 2 près famille \\
\hline & & & & 1 bact 4 & 0 loin famille \\
\hline \multirow[t]{3}{*}{$\begin{array}{l}\text { Grande exploitation } \\
\text { agricole }\end{array}$} & 8 & de $4 \mathrm{a}+$ de 10 & 0 & 4 bac & 3 dans famillc \\
\hline & & & & 3 bac +2 & 3 près famille \\
\hline & & & & 1 bact 4 & 2 loin famille \\
\hline \multirow[t]{3}{*}{ Fonctionnaire moyen } & 6 & de $1 \mathrm{a} 4$ & 0 & $1 \mathrm{bac}$ & 4 dans famillc \\
\hline & & & & 3 bact 2 & 2 près famille \\
\hline & & & & 2 bac +4 & \\
\hline \multirow[t]{3}{*}{ Haut fonctionnaire } & 9 & de $1 \mathrm{a} 3$ & 2 & 2 bac & 5 dans famille \\
\hline & & & & 3 bac +2 & 3 près famille \\
\hline & & & & 4 bac 44 & 1 loin famille \\
\hline $\begin{array}{l}\text { Profession libérale } \\
\text { supérieure }\end{array}$ & 11 & de 2 a 9 & 2 & 1 bac & 8 dans famille \\
\hline \multirow[t]{2}{*}{ Avocats, médecins, ... } & & & & 4 bac +2 & 2 près famille \\
\hline & & & & 6 bac 44 & 1 loin famille \\
\hline \multirow[t]{3}{*}{ Ouvrier employé en ville } & e 14 & de 3 à 10 & 1 & 2 bac & 7 dans famille \\
\hline & & & & 7 bact 2 & 5 près famille \\
\hline & & & & 5 bac +4 & 2 loin famille \\
\hline \multirow[t]{3}{*}{$\begin{array}{l}\text { Artisan, contremaitre } \\
\text { en ville }\end{array}$} & 8 & de $2 \mathrm{a}+$ de 10 & 1 & 4 bac & 2 dans famille \\
\hline & & & & 2 bac +2 & 3 près famille \\
\hline & & & & 2 bac +4 & 3 loin famille \\
\hline \multirow[t]{3}{*}{ Commerçant en ville } & 11 & de 1 a 10 & 0 & 4 bac & 5 dans famille \\
\hline & & & & 6 bact 2 & 2 près famille \\
\hline & & & & 1 bact4 & 4 loin famille \\
\hline TOTAL & 79 & de $1 \mathrm{a}+\mathrm{de} 10$ & 6 & $23 / 33 / 23$ & $41 / 24 / 14$ \\
\hline
\end{tabular}

Ce tableau permet de constater :

- La longue présence, dans les villes ou villages du Roussillon et de Catalogne espagnole des familles des jeunes trafiquants : sur 79 familles, 68 résident dans les mêmes lieux depuis plus de deux générations, 53 depuis plus de quatre générations et 30 depuis plus de sept générations ;

27 - Les plus anciennes familles sont celles qui appartiennent aux milieux les plus modestes, propriétaires de petites exploitations familiales agricoles, ouvriers et employés de Perpignan, Gérone, Lérida, Barcelone, Tarragone, mais aussi les plus favorisés, avocats, médecins, notaires, grands exploitants agricoles, et encore intermédiaires, artisans, contremaîtres et commerçants ; les fonctionnaires, malgré leur mobilité sont « du lieu » parfois depuis trois ou quatre générations.

Il s'agit donc bien de familles « d'ici », de celles qui font référence, qui participent à la production et à la conservation des valeurs, des normes, qui savent énoncer l'ordre des légitimités locales, faire référence à cet «âge d'or » qui leur confère une forte influence sociale (Halbwachs, 1937). On pourrait supposer qu'il s'agit d'une activité en quelque sorte "réservée » aux fils de familles qui ont "mal tourné », qui sont en rupture : il n'en est rien, plus de la moitié de notre échantillon (52\%) de jeunes trafiquants partage le domicile des parents, et $30 \%$ habitent à proximité, dans le même quartier ou le même village (Grafmeyer et Dansereau, 1998 ; Lévy, 1998). On peut penser aussi à des mises en difficulté par des échecs scolaires ou universitaires? ce n'était pas le cas, puisque $70 \%$ d'entre eux ont au moins le niveau bac plus deux et $30 \%$ le niveau bac plus quatre au moins; sept jeunes sur neuf, enfants de hauts fonctionnaires (ingénieurs, administrateurs, professeurs d'université) ont une formation supérieure à bac plus deux (et la plupart de ces jeunes trafiquants étant en cours d'étude, ces qualifications ne feront que s'accentuer). Douze enfants d'ouvriers et employés en milieu urbain sur quatorze ont acquis, au moment de notre enquête, un niveau d'études 
supérieur à bac plus deux, comme cinq enfants sur sept d'ouvriers et d'employés résidant en milieu rural. Faut-il dès lors chercher auprès des parents quelque événement provoquant une rupture de ces continuités morales et territoriales locales? Les divorces ne concernent que 7,5\% des familles de nos jeunes trafiquants, c'est-àdire qu'ils sont très nettement inférieurs aux moyennes nationales. En somme, si nous devions proposer un portrait type du jeune trafiquant, nonconsommateur, nous dirions qu'il s'agit d'un jeune homme poursuivant des études supérieures, attaché à sa famille, unie et présente depuis plusieurs générations dans le lieu. Un jeune qui fait continuité familiale et non pas un "héritier malheureux " (Gotman, 1994). Nous sommes au cœur de milieux marqués par la conservation et non par la rupture.

ous avons pu tracer vingt-trois généalogies à trois ou quatre niveaux générationnels, incluant des lignées parentales proches ; trois « modèles » se dégagent et suggèrent que nous avons à faire dans la quasi-totalité des cas à des familles en ascension sociale, culturelle et économique, y compris dans les milieux ouvriers, et rien ne semble différencier les places et trajectoires de ces jeunes de leurs cousins proches ou plus lointains : rien, sinon cette activité invisible des trafics de psychotropes pratiquée sur le mode d'une activité lucrative, souvent transitoire.

Cohésions sociales locales et stratégies de « sortie » des jeunes des « carrières de trafiquants "

31 La cohésion familiale autorise à développer des stratégies de "sortie », lorsque le besoin se fait sentir, qui sont généralement efficaces auprès des autorités judiciaires et policières locales parce que facilitées, portées, par une société de «braves gens » bien connus.

Il convient de remarquer que les moyens d'une "mise à distance " par une fuite, une nouvelle localisation du jeune trafiquant est souvent hors de portée des familles qui sont «d'ici » et n'entretiennent que peu de réseaux géographiquement lointains. La « distance sociale » par contre est facile à /r/établir entre le jeune et le milieu des trafiquants professionnels "permanents » qui le fournissent en psychotropes divers: l'invisibilité du jeune « fils de famille » tient à cette extranéité même. Le jeune est donc absorbé par la densité du lien social familial et local : stages chez un oncle, si le manque d'emploi est présenté comme cause de la délinquance, sorties avec les cousins ou autres parents collatéraux, si l'ennui, la solitude tient lieu d'excuse. En outre ces familles sont localement à l'intersection de nombreuses associations, groupes d'affinité, etc., qui sont des ressources utiles. Les "sorties" sont en quelque sorte simples et immédiates. Le jeune était « sorti » de la famille et de ses exigences de représentativité locale, et voici tout simplement qu'on le fait à nouveau " entrer » dans le rang. Cela ressemble fort aux voyages vers l'Inde des jeunes en rupture de banc familial dans les années 1970. La banalisation de ces circuits est telle que, dans un cas, une sœur reprend immédiatement le commerce de psychotropes abandonné par le jeune découvert. Des amis, tout aussi "propres", demandent même, lorsqu'ils sont dans la confidence, à bénéficier de cette situation lucrative de passeur. Quant aux Gitans, ce sont des interlocuteurs bien commodes : pourvu qu'un semblable « invisible » remplace celui qui réintègre son milieu et sa morale sociale, il n'y a pas de problème :

«On les voit défiler de loin, nous, ce qu'on leur dit, c'est si vous pouvez pas, un jeune comme vous doit vous remplacer, un «invisible» en vaut un autre. De toute façon quand ils arrêtent on ne les voit plus pendant quelques jours ou un peu plus 
et puis tu les vois rappliquer pour demander leur "caution" ; alors, on règle tout, bien, bien » (gitan domicilié à Perpignan). chargements importants de cocaïne et d'hérö̈ne, mais encore d'anabolisants, en direction de la frontière italienne ; entre Toulon et Nice la 'marchandise' est livrée à un passeur en route pour l'Italie ${ }^{11}$. Il leur est strictement interdit de négocier tout ou partie des substances transportées avec des dealers locaux tout au long de leur trajet. Des jeunes trafiquants de «bonnes familles » effectuent chaque mois ces transports; d'autres une fois par trimestre ou moins. Dès lors que tel ou tel est arrêté à la frontière, particulièrement fouillé, suspecté donc, de tels transports ne lui sont plus confiés. Souvent les passages de frontière sont effectués par un couple, réel ou fictif, accompagné d'un enfant d'amis ou de parents. Ces trajets visent à libérer des Italiens des activités de passeurs, qui devenaient de plus en plus visibles. La présentation de soi sous forme de "jeune famille convenable " était déjà utilisée par les Italiens, qui n'hésitaient pas à effectuer des allers-retours en camping car.

- Les ventes aux consommateurs «socialement proches». Il s'agit en fait d'un secteur d'activité de haute rentabilité : celui des consommateurs que le jeune peut toucher ou les personnes qu'il peut amener à consommation d'un quelconque psychotrope d'usage illégal, du fait de sa propre position sociale. Le principe de cohésion sociale joue à plein : d'une part l'influence de ces jeunes de bonnes familles est importante dans leur milieu proche, et d'autre part les transactions sont protégées par le caractère intragroupal de ces affinités ${ }^{12}$.

37 - Les ventes aux personnes originaires d'autres lieux et d'autres milieux, qui sont orientées vers tel ou tel jeune par le "correspondant Gitan local», par crainte de repérage, ou pour accélérer les rotations transfrontalières du jeune. Cette dernière activité est à la fois crainte, car ces nouveaux venus visibilisent le jeune trafiquant, et désirée car il s'agit là de gains faciles ${ }^{13}$. Certaines personnes, des jeunes femmes notamment, se spécialisent dans cette troisième pratique à partir d'activités de vente commerciale légale dans des magasins ou des véhicules.

Parmi les quinze jeunes trafiquants qui nous signalèrent avec précision les gains de l'année précédent notre rencontre (1997 pour la plupart), les bénéfices allaient de 128058 euros à 350633 euros.

Nous avons pu connaître cinq personnes, dans le département des Pyrénées-orientales, qui développent, à partir de leurs gains dans les trafics de psychotropes, des réussites professionnelles certaines mais bien d'autres personnes, couples ou familles, connaissent probablement de telles situations de dissimulation et de réussite économique à partir des activités de trafics transfrontaliers. Il faut systématiquement multiplier par 3 ou 4 les chiffres que nous signalons pour restituer une proportion

Revue européenne des migrations internationales, vol. 18 - $n^{\circ} 3$ | 2002 
correspondante aux désignations faites par les personnes rencontrées; ainsi, lorsque nous connaissons six personnes qui semblent à l'abri de tout soupçon (novembre 1998), il faut compter une cohorte de 20 à 25 personnes dans cette situation dans le département des Pyrénées-orientales. On peut donc penser qu'un peu moins de 20 personnes investissement, dans leur réussite professionnelle, des sommes gagnées dans les trafics. En fait ce nombre doit être majoré de ceux, beaucoup plus nombreux qui, soupçonnés ou pris, rejoignent leur famille. Bien sûr ils ne poursuivent pas leurs activités, mais tout de même disposent dans la plupart des cas de plusieurs centaines de milliers de francs. Nous pouvons raisonnablement situer autour de quatre-vingts personnes dans les seules Pyrénées-orientales la population qui, depuis 1996, a pu tirer bénéfice de ses trafics pour sa réussite personnelle dans des domaines d'activités légales.

La jeune femme de 26 ans que nous avons rencontrée à Lérida blanchit son argent dans une activité commerciale légale; elle paie la location de sa boutique, un salaire d'employée et les rotations de stocks avec les bénéfices de ses voyages jusqu'à réussite de son activité :

«Avant j'y allais toutes les semaines, puis j'ai commencé le commerce, et maintenant j'en suis au maximum à un voyage par mois. Bientôt j'irai vraiment en cas de besoin. Ce que je vends se paie surtout en liquide, alors pas de problème ; ma seule astuce c'est de déclarer plus d'achats que j'en fais réellement. Et c'est très facile; d'habitude c'est l'inverse qu'on demande à nos fournisseurs [...]. Alors, j'ai des fournisseurs qui passent du black à d'autres commerçants et qui sont tout heureux de trouver ma pomme pour blanchir leurs affaires. Dix pas déclarés pour $\mathrm{X}$, et dix déclarés en trop pour moi ».

Pour un jeune agriculteur, rencontré à Gérone et à Tarragone, la maison familiale a été magnifiquement réhabilitée, au noir, et des membres de la famille sont censés vendre régulièrement sur de nombreux marchés : cette activité est quasi imaginaire, mais permet de justifier de confortables rentrées en liquide. Les ouvriers agricoles qui travaillent dans l'exploitation sont déclarés («je vais pas tomber pour une connerie comme ça; et puis ces gens ont leur dignité »). Par ailleurs cette personne nous dit " placer de l'argent en Espagne ».

\section{Conclusion}

Cette recherche révèle un double phénomène: l'un concernant l'apparition de trafiquants non-consommateurs parmi les jeunes de la «bourgeoisie » locale au sud et au nord de la frontière franco-espagnole et l'autre qui lui est concomitant permet de voir une sorte de microlocalisation-banalisation de leurs activités à partir d'incitations en provenance des milieux Gitans catalans et andalous. C'est donc, le savoir circuler, passer les frontières, faire unité territoriale grâce à l'expansion des clans qui place les Gitans "étrangers de l'intérieur", peu sédentarisés en situation de maitrise commerciale dans les divers lieux du trafic. Ces mêmes Gitans, dans ces vastes espaces, s'entendirent d'abord avec les milieux traditionnels, interlopes, des trafics, ceux qu'on appelle les "voyous locaux", se placèrent sur le marché des consommations des prostitué(e)s, et créèrent des réseaux de dealers nouveaux : des jeunes gens de familles depuis longtemps stabilisées dans les villages et villes de leur « territoire commercial». Ainsi ils ajoutaient encore un ordre et une efficacité au désordre des livraisons intermédiaires qui existaient auparavant (avant 1996).

L'entrée de ces « jeunes de bonnes familles » dans les trafics transfrontaliers de drogues nous montre aussi comment se généralisent et se banalisent rapidement les activités 
des trafiquants de psychotropes : ce n'est plus le repliement, l'étanchéité classique des contours familiaux mafieux qui, le long de réseaux ainsi bien protégés, mais à distance des sociétés locales facilitent la circulation des psychotropes interdits; c'est au contraire la forte insertion dans des milieux sociaux locaux de haute cohésion et porteurs des valeurs les plus conservatrices qui facilite, protège, habilite ces carrières de trafiquants. Même si ces valeurs ne sont attachées qu'à la réussite économique, nonobstant les causes et effets dangereux des consommations liées à ces trafics pour les personnes et pour les sociétés. Pourrait-on tout au plus avancer l'idée que la ville a désormais envahi tous les recoins de nos sociétés, et ainsi que l'anonymat (Simmel, 1984) qui lui est lié dissout la vigueur et la prégnance des exemplarités familiales. Mais ce type d'explications par les attributs du creuset urbain se révèle incapable de préciser le personnage du communautaire, villageois au cœur de la ville. C'est peut-être là une rançon liée aux nombreux effets de métissages contemporains.

\section{BIBLIOGRAPHIE}

BOUHNIK Patricia (1994) Le monde social des usagers de drogue dure en milieu urbain défavorisé, Thèse de doctorat de sociologie, Université Paris 8.

BOURGOIS Philippe (1995) In search of respect; selling crack in El Bario. Cambridge, University press.

DUPREZ Dominique, KOKOREFF Michel et VERBEKE A. (1995) Des produits aux carrières : contribution à une sociologie du trafic des stupéfiants, Lille, CLERSE, IFRESI, Rapport de recherche.

DUPREZ Dominique et KOKOREFF Michel (2000) Les mondes de la drogue, Paris, Éd. Odile Jacob.

EHRENBERG Alain (1998) Drogues et médicaments psychotropes, le trouble des frontières, Paris, Esprit.

GOTMAN Anne (1994) Dilapidation et prodigalité, Nathan, Essais et Recherches.

GRAFMEYER Yves et DANSEREAU F (dir) (1998) Trajectoires familiales et espaces de vie en milieu urbain, Presse Universitaire de Lyon.

HALBWACHS Maurice (1937) La mémoire collective, Presse Universitaire de France.

HARVEY David L. (1993) Potter Addition, Poverty, Family and Kingship in a heartland community, New York, Aldine de Gruyter.

HILY Marie-Antoinette (2001) Rencontres Interculturelles : Échanges et Sociabilités, in Roselyne de Villanova, Marie-Antoinette Hily et Gabrielle Varro Éds., Construire l'interculturel ? De la notion aux pratiques, Paris, l'Harmattan, pp.7-17.

LEVY J.P (1998) Habitats et habitants : position et mobilité dans l'espace résidentiel, Presse Universitaire de Lyon, coll. Transversales.

MISSAOUI Lamia et TARRIUS Alain (1999) Jeunes de bonnes familles dans les trafics frontaliers d'hérö̈ne, de Barcelone à Perpignan, Montpellier et Toulouse, Éd. Trabucaire, coll. Recherches en cours. Perpignan, Barcelone. 
MISSAOUI Lamia (1999) Gitans et santé : de Barcelone à Turin, les compétences de l'étranger de l'intérieur ; ethnicité et métissage chez les Gitans catalans et andalous autour des problèmes de santé publique, Trabucaire.

POUTIGNAT Philippe et STREIFF-FENART Jocelyne (1995) Les théories de l'ethnicité, Presse Universitaire de France.

RÉMY Jean (1995) Simmel, ville et modernité, Paris, L'Harmattan.

RINAUDO Christian (1998) La construction sociale de l'ethnicité en milieu urbain, Thèse de Doctorat, Université de Nice.

SIMMEL Georg (1984) Métropoles et mentalités, in Yves Grafmeyer et Isaac Joseph, l'École de Chicago, Naissance d'une écologie urbaine.

\section{NOTES}

1. -Les Gitans catalans de Perpignan et de Barcelone accédèrent à l'héroïne en même temps que les populations barcelonaises non gitanes : autour de l'année 1980. L'Espagne vivait alors sa construction de jeune démocratie dans une certaine allégresse ; les milieux « contre-culturels », qui exprimaient au plus haut point les exigences d'alternatives aux constructions sociales franquistes et qui n'étaient donc pas dans ces circonstances mis ou maintenus en marge, comme ailleurs en Europe, passèrent massivement à la consommation de divers psychotropes dont la cocaïne et l'héroïne. Les Gitans jouèrent à l'époque un rôle d'associés emblématiques : ils furent présentés comme ceux qui avaient toujours su résister au tarissement culturel franquiste, comme des associés, des compagnons de route naturels dans cette recherche d'alternatives. Nombre de liaisons entre jeunes Gitans et filles de la bourgeoisie barcelonaise s'exposèrent alors comme transgression des cloisonnements anciens. La consommation faisait partie des attributs de la transgression. Des jeunes Gitans de Perpignan, dont les familles n'avaient jamais rompu les liens avec leurs parents de Barcelone, participèrent à ce mouvement.

2. -Pour G. Simmel, la forme n'est pas qu'apparence, son approche conduit le chercheur à l'analyse des trans-formations du social (Rémy, 1995).

3. -Une tendance actuelle de la recherche en sciences sociales, concernant les trafics et les consommations de psychotropes désigne les groupes ethniques comme porteurs de comportement spécifiques et justifie ainsi la recherche au cœur de ces groupes ou des communautés. Les nations anglo-saxonnes, qui admettent constitutionnellement le fait communautaire ethnique, au contraire de la France, sont particulièrement concernées par ces positions de recherches.

4. -Un exemple caractéristique de ces façons du chercheur inspiré par les thèses de Barth peut se lire dans la thèse de Rinaudo (1998).

5. - « Paio » terme utilisé par les Gitans pour désigner ceux qui ne sont pas Gitans.

6. -En effet, jusqu'en 1996 encore, la distribution des psychotropes était affaire de réseaux spécialisés dans tel ou tel produit ; Barcelone apparaissait comme place centrale de concentration avant diffusion, et les consommateurs d'espaces d'abord voisins puis de plus en plus éloignés concentriquement venaient prélever ; des dealers ou voyous notoires, de diverses origines locales, étaient impliqués dans ces trafics ; diverses filières aboutissaient donc à Barcelone, sans apparents problèmes de rivalités commerciales et là vendaient la totalité ou une partie de leurs apports ; deux grandes 
filières se distinguaient davantage, celle de l'héroïne d'origine presque exclusivement nigériane (avec diverses modalités de mise en réseau, et probablement des fins de fabrication dans la région de Grenade et au Maroc), et celle de la cocaïne d'origine latino-américaine, transitant par le Portugal. Des Sénégalais et Gambiens participaient alors au contrôle et à la diffusion de l'héroöne (une part majeure transitait vers d'autres pays, une autre part irriguait le Nord de l'Espagne et le Sud de la France, et enfin une part très mineure était réservée aux consommations locales selon les modes de distribution concentriques signalées). L'ecstasy et les amphétamines étaient peu visibles, quant au cannabis et ses nombreux dérivés, les remontées du Maroc satisfaisaient le marché suivant divers modes (économies souterraines des " fourmis » des trafics internationaux, bateaux et camions).

7. -On trouve des profils semblables dans les descriptions de Harvey (1993).

8. -Nous qualifions d'échantillon la somme des personnes aux profils conformes à l'objet de notre recherche, que nous avons pu rencontrer au cours de nos enquêtes de terrain. La significativité de cet échantillonnage réside dans l'existence même d'une population qui apparaît à partir de 1995 et va se densifiant. Bien sûr la base d'échantillonnage n'existe pas, mais nous pouvons affirmer que 79 personnes identifiées et rencontrées en 1997 et 1998 réalisent très probablement un échantillon significativement représentatif au sens statistique du terme. Il suffit en effet de partir de cette cohorte et de faire varier les indices de confiance pour se rendre compte que 79 personnes représentent une proportion significative pour une population de plusieurs centaines de personnes. Nous sommes probablement très au-delà des $10 \%$ de représentativité : huit cents nouveaux trafiquants, organisés en nouveaux réseaux de distribution en Catalogne seule se remarqueraient fort dans le paysage si observé, surveillé, de ces activités. Empiriquement, à partir des déclarations de nos 79 interlocuteurs, nous sommes enclin à situer autour de 400 à 450 personnes la totalité de ces nouveaux entrepreneurs, dont une centaine pour la partie française. Il s'agit bien sûr de la somme des personnes en activité et de celles qui ont cessé de s'impliquer dans ces trafics (prison, arrestations, réactions des familles...).

9. - La « caution » est une institution caractéristique de ces filières : au bout de quatre ou cinq passages réalisés sans embûches (gain d'environ 38 à 60980 euros pour les transports - qui peuvent parfois nécessiter une mobilité jusqu'à la frontière italienne -, indépendamment des reventes « personnelles » directes à des consommateurs) le fournisseur de Lérida, de Gérone ou de Tarragone, prélève une somme de 13 à 15245 euros sur ses dettes envers le jeune trafiquant (il y a « dette » du fournisseur car le jeune trafiquant, après deux voyages, doit faire l'avance du prochain chargement, ou du moins d'une grande partie) et lui signifie qu'en « fin de travail » tel parent à lui, résidant à Perpignan, la lui rendra. Cet allié d'un même clan devient dès lors le correspondant local du jeune de «bonne famille».

10. -À l'exception de l'un d'entre eux qui, après une détention de huit mois, s'engagea comme logisticien dans une $\mathrm{ONG}$ en zone subsaharienne tout en rompant avec sa famille, le plus grand nombre consolidait ses relations avec les milieux gitans andalous, avec les Noirs-africains en migration, et souvent avec des marocains spécialisés dans les transports de psychotropes (résine de cannabis mais aussi cocaïne, héroïne, etc.). Ils entrent alors dans un univers nouveau pour eux : accueillis et hébergés par ces " compagnons ", ils deviennent des voyageurs internationaux prêts à toutes les astuces pour acquérir une notoriété nouvelle.

11. -Un tel voyage peut rapporter jusqu'à 15245 euros. 
12. -Un trafiquant « moyen » recueille mensuellement dans cette activité environ 12196 euros de bénéfice, un " grand » de 18294 à 22867 euros et un « petit » de 4574 à 6098 euros.

13. -Les bénéfices de cette troisième activité sont de l'ordre de 4574 euros par mois.

\section{RÉSUMÉS}

Cet article relate l'apparition d'une nouvelle forme sociale caractérisée par l'entrée des " jeunes de bonnes familles" dans les trafics transfrontaliers de drogues, à partir d'incitations en provenance des milieux gitans catalans et andalous. Cela nous montre comment se généralisent et se banalisent les activités des trafiquants de psychotropes : ce n'est plus le repliement, l'étanchéité classique des contours familiaux mafieux qui, le long de réseaux ainsi bien protégés, mais à distance des sociétés locales, facilitent la circulation des psychotropes interdits ; c'est la forte insertion dans des milieux sociaux locaux de haute cohésion et porteurs des valeurs les plus conservatrices qui facilite, protège et habilite ces carrières de trafiquants.

Gypsies and Youth from "Good Families" in the Drug Traffic. -- This article present a new social form characterized by the entry of youth from "good families" in the trans-frontier traffic in drugs, under the influence of Catalan and Andalusian Gypsies. This shows us how quickly the activities of narco-traffickers expand and become normalized; they are no longer closed-off and clearly demarcated by Mafioso family contours, where prohibited psychotropic substances circulate through networks that are well protected but at a distance from local societies. Rather, these activities are well implanted in local social milieux that are highly cohesive and associated with very conservative values, and where the careers of traffickers are facilitated, protected and enabled.

Gitanos y jóvenes de "buenas familias" en el trafico de drogas. -- El articulo relata la aparición de una nueva forma social, caracterizada por la aparición de "jóvenes de buenas familias", en el trafico fronterizo de drogas a partir de las incitaciones de los gitanos catalanes y andaluces. El autor nos muestra como se generalizan y se banalizan las actividades de traficantes de psicótropos: ya no se trata del repliegue clásico en los limites de la familia de la mafia quien a través de sus redes protegidas pero a distancia de las sociedades locales facilita la circulación de los psicótropos prohibidos : se trata de la fuerte inserción en los medios sociales locales de gran cohesión y portadores de los valores mas conservadores lo que facilita, protege y habilita el desarrollo de verdaderas carreras de traficante.

\section{INDEX}

Mots-clés : frontière, Gitans, jeunes, toxicomanie

Index géographique : Espagne, France 


\section{AUTEUR}

\section{LAMIA MISSAOUI}

Maître de Conférences à l'Université de Versailles St-Quentin-en-Yvelines, Laboratoire PRINTEMPS, 47 Boulevard Vauban, 78047 Guyancourt Cedex. 